\title{
Long-range correlation studies at the SPS energies in MC model with string fusion
}

\author{
Vladimir Kovalenko* \\ Saint Petersburg State University \\ E-mail: nvkinferambler.ru \\ Vladimir Vechernin \\ Saint Petersburg State University \\ E-mail: vechernin@gmail.com
}

\begin{abstract}
Studies of the ultrarelativistic collisions of hadrons and nuclei at different centrality and energy enable to explore the QCD phase diagram in a wide range of temperature and baryon density. Long-range correlation studies are considered as a tool, sensitive to the observation of phase transition and the critical point. In the present work, a Monte Carlo model of proton-proton, proton-nucleus, and nucleus-nucleus collisions is applied to heavy and light ion collisions at the cms energy range from a few up to several hundred $\mathrm{GeV}$ per nucleon. The model describes the nuclear collisions at the partonic level through interaction of color dipoles and takes into account the effects of string fusion, which can be considered as an alternative to relativistic hydrodynamics way of describing the collective phenomena in heavy-ion collisions. The implementing of both the string fusion and the finite rapidity length of strings allowed to consider the particle production at non-zero baryochemical potential. We calculated the long-range correlation functions and correlation coefficients between multiplicities and transverse momentum at several energies for different colliding systems and obtained predictions for the experiment.
\end{abstract}

XXII International Baldin Seminar on High Energy Physics Problems,

15-20 September 2014

JINR, Dubna, Russia

${ }^{*}$ Speaker. 


\section{Introduction}

The exploration of the phase diagram of strongly interacting matter and a search for the onset of deconfinement and the critical point is one of the main subjects of heavy ion physics. There is a general consensus that at zero baryochemical potential the deconfinement phase transition is a smooth cross-over [1], which is mainly based on the lattice QCD calculations. However, the numerical lattice study of the behaviour of the QCD matter at non-zero baryon density, due to the sign problem, is not straightforward [2, 3, 4]. According to existing calculations [5, 6, 7] it is expected that at high net baryon density, this transition is of the type of the first order, which suggests an existence of a critical point in the phase diagram at the intermediate net-baryon density. However, in several works a smooth crossover has been obtained at any value of baryochemical potential $[8,9]$.

The experimental investigations on the QCD phase diagram are related to the study of the collisions of the ions at high energy $[10,11]$. The study of the QCD phase diagram is a part of physical program of the NA61 experiment at the SPS, experiments at RHIC and also future detectors CBM at FAIR and MPD at NICA [12, 13, 14, 15]. Among the observables, being used in these studies, the most sensitive ones, such as a collective flow, correlations, fluctuations, require event-by-event analysis $[16,17]$. Particularly, the studies of long-range correlations between variables taken from two different rapidity windows are included in the research program of the experiment NA61 as a tool, sensitive to the observation of phase transition and the critical point [17].

For the correct interpretation of experimental findings and evaluation of the sensitivity of experimental methods, the theoretical modelling of the evolution of a heavy-ion collision with the explicit calculation of observables in the conditions, close to the experimental ones, is required. Due to the complexity of the evolution process of a heavy-ion collision and inapplicability of QCD perturbation theory in the low momentum region, semiphenomenological models are widely applied in this area.

One of the models, which is used to describe the properties of the initial dense state of the strongly interacting matter that occurs immediately after the nuclear interaction of high energy, is the model for the formation and fusion of quark-gluon strings [18, 19, 20, 21, 22]. According to this model, the hadrons, produced in the soft region, can be described as a result of decay of colour field tubes - strings, which are formed between the interacting partons. With increasing energy and the mass number of colliding nuclei, the density of strings grows, and they begin to overlap forming an area in a transverse plane with stronger color field ("clusters"). In the limit of high density, it is expected that all cross-section plane will be a single cluster, which is supposed to be in its physical properties equivalent to the quark-gluon plasma. Convincing evidence in favour of the fusion of the strings are discovered experimentally growth of the mean transverse momentum with multiplicity in hadron and nuclear collisions and a significant decrease in the yield of multiplicity in the collisions of heavy ions compared to models with independent strings, which is compatible with the existing experimental data on multiplicity. It is assumed that fusion and percolation of strings responsible for the appearance of the ridge in two-particle correlations [23, 24]. In the recent papers $[25,26,27]$ it was shown that the equation of state of QGP at zero chemical potential obtained in the colour string percolation model is in an excellent agreement with the lattice results. In addition, the approach has been successfully applied for the determination of the shear viscosity 
[28] of QCD matter, with was found in a good agreement with experimental data in a wide range of temperatures.

In the framework of the string fusion approach, the critical behaviour is expected when the processes of string fusion and percolation come into play, what can be considered as a possible way of quark-gluon plasma formation. Around percolation threshold, strong fluctuations in colors of strings appear what lead to large fluctuations in some observables, which one can find by the event by event analysis [17]. In the present work, the model is applied to heavy and light ion collisions at the center of mass energy range from a few up to several hundred $\mathrm{GeV}$ per nucleon. In the next sections, we very briefly describe the main features of the Monte Carlo model with string fusion and explain how it can be applied at non-zero baryon density. Then, we introduce the observables (correlation functions and correlation coefficients) used in the study. Finally, we present the results and give our conclusions.

\section{The Monte Carlo model}

The present model $[29,30]$ describes nuclear collisions on the partonic level, without referring to the Glauber picture of the independent collisions of the nucleons. Initially, the nucleons are supposed to be distributed in a nucleus according to the charge density distribution $\rho(r)$. We used harmonic oscillator model for ${ }^{7} \mathrm{Be}$ and ${ }^{9} \mathrm{Be}$ nuclei and Woods Saxon distribution for heavier species [31].

\begin{tabular}{|c|c|c|c|}
\hline Nucleus & Model & $\rho(r)$ & Parameters \\
\hline${ }^{208} \mathrm{~Pb}$ & Woods Saxon & $\frac{\rho_{0}}{1+\exp [(r-R) / d]}$ & $R=6.63 \mathrm{fm}, d=0.545 \mathrm{fm}$ \\
${ }^{197} \mathrm{Au}$ & Woods Saxon & $\frac{\rho_{0}}{1+\exp [(r-R) / d]}$ & $R=6.38 \mathrm{fm}, d=0.535 \mathrm{fm}$ \\
${ }^{40} \mathrm{Ca},{ }^{40} \mathrm{Ar}$ & Woods Saxon & $\frac{\rho_{0}}{1+\exp [(r-R) / d]}$ & $R=3.53 \mathrm{fm}, d=0.542 \mathrm{fm}$ \\
${ }^{7} \mathrm{Be}$ & Harmonic oscillator & $\rho_{0}\left(1+a\left(r / r_{0}\right)^{2}\right) \exp \left(-\left(r / r_{0}\right)^{2}\right)$ & $r_{0}=1.77 \mathrm{fm}, \mathrm{a}=0.327$ \\
${ }^{9} \mathrm{Be}$ & Harmonic oscillator & $\rho_{0}\left(1+a\left(r / r_{0}\right)^{2}\right) \exp \left(-\left(r / r_{0}\right)^{2}\right)$ & $r_{0}=1.791 \mathrm{fm}, \mathrm{a}=0.611$ \\
\hline
\end{tabular}

Table 1: Nuclear distributions and parameters used in the present study.

In the model, each nucleon contains a valence quark-diquark pair and several sea quarkantiquark pairs. The number of sea pairs is generated according to Poisson distribution around some mean value, which is energy-dependent and is adjusted to describe the total inelastic cross section in pp collisions. The transverse coordinates of these partons are distributed around the center of each nucleon according to two-dimensional Gauss distribution with a mean-square radius $r_{0}$. 
It is assumed that quark-diquark and quark-antiquark pairs form dipoles. An elementary interaction according to the color dipole approach $[32,33]$ is realized with the probability amplitude given by:

$$
f=\frac{\alpha_{S}^{2}}{2}\left[K_{0}\left(\frac{\left|\vec{r}_{1}-\vec{r}_{1}^{\prime}\right|}{r_{\max }}\right)+K_{0}\left(\frac{\left|\vec{r}_{2}-\vec{r}_{2}^{\prime}\right|}{r_{\max }}\right)-K_{0}\left(\frac{\left|\vec{r}_{1}-\vec{r}_{2}^{\prime}\right|}{r_{\max }}\right)-K_{0}\left(\frac{\left|\vec{r}_{2}-\vec{r}_{1}^{\prime}\right|}{r_{\max }}\right)\right]^{2}
$$

where $K_{0}$ is modified Bessel function of the second order, $\left(\vec{r}_{1}, \vec{r}_{2}\right),\left(\vec{r}_{1}^{\prime}, \vec{r}_{2}^{\prime}\right)$ are transverse coordinates of the projectile and target dipoles, and $\alpha_{s}$ - effective coupling constant, $r_{\max }$ is characteristic confinement scale. According to this formula, two dipoles interact with higher probability, if the ends of dipoles are close to each other in the transverse plane, and (other equal) if they are wide.

In our Monte Carlo model it is assumed, that if there is a collision between two dipoles, two quark-gluon strings are stretched between the ends of the dipoles, and the process of string fragmentation gives observable particles. The particle production of a string is assumed to go uniformly between the string rapidity ends $y_{\min }$ and $y_{\max }[29,34]$, which are related to the longitudinal momentum fraction, carried by the parons forming the string. The emission of the charged particles is generated with mean number per rapidity $\langle\mu\rangle$, independently in each rapidity interval, with Poisson distribution.

The transverse position of string is assigned to the arithmetic mean of the transverse coordinates of the partons at the ends of the string. Due to finite transverse size of the strings they overlap, that in the framework of string fusion model $[18,19]$ gives the source of a higher tension. We used the lattice variant of the model $[35,36]$, according to which strings are supposed to be fused if their centres occupy the same cell. The area of each cell is equal to the transverse string area $\left(S_{\text {str }}=\pi r_{\text {str }}^{2}\right)$. In the calculations, we also assume local string fusion scenario [35, 36]. Each bin in rapidity with an integer number of overlapped strings is processed separately. Mean multiplicity of charged particles and mean $p_{t}$ originated from the cell where $k$ strings are overlapping are obtained according the following:

$$
\langle\mu\rangle_{k}=\mu_{0} \sqrt{k}, \quad\left\langle p_{t}\right\rangle_{k}=p_{0} \sqrt[4]{k} .
$$

Here $\mu_{0}$ and $p_{0}$ are mean charged multiplicity from one single string per rapidity unit and mean transverse momentum from one single string. The relations allows to calculate long-range correlation functions and correlation coefficients between multiplicities and mean transverse momentum of charged particles [30]. Important, that $\mu_{0}$ and $p_{0}$ cancels out in the correlation observables defined below and do not influence the final result on correlation coefficients, which makes the calculations more robust.

The parameters of the model $\alpha_{s}, r_{0}, r_{\max }, \mu_{0}$ are supposed to be energy-independent and have been constrained from charged particles multiplicity in a wide energy range (from ISR to LHC) in pp collisions and in minimum bias $\mathrm{p}-\mathrm{Pb}$ and $\mathrm{Pb}-\mathrm{Pb}$ collisions at the LHC energy. The detailed procedure of parameters tuning is described in [34].

Important to note, that the implementation of the string fusion in the model, described above, allows us to separate phenomena taking place (in the same event) in different rapidity regions. At the SPS energies, the contribution of sea quarks and corresponding strings is not significant. Therefore, the valence strings dominate. Due to the fact that the diquark carries out larger longitudinal momentum fraction [29], the valence string, formed by a quark-diquark pair, is asymmetric. Most of the particles in forward rapidity region are coming from valence strings from their diquark parts. 
The approximate particle content of the valence rapidity string is shown in Fig. 1. The diquark end of the string, which is generally located in the forward rapidity region, is characterized by large baryon yield. In contrary, the opposite end, which carries out a quark, emits mostly pions, which happens around midrapidity. Altogether, it provides a distribution of a net-baryon density over rapidity with large baryon redundancy in the forward region, which has been obtained in the experimental data (Fig. 2). We note, that the valence string model, developed in [37], successfully describes net baryon yields in a wide energy range.

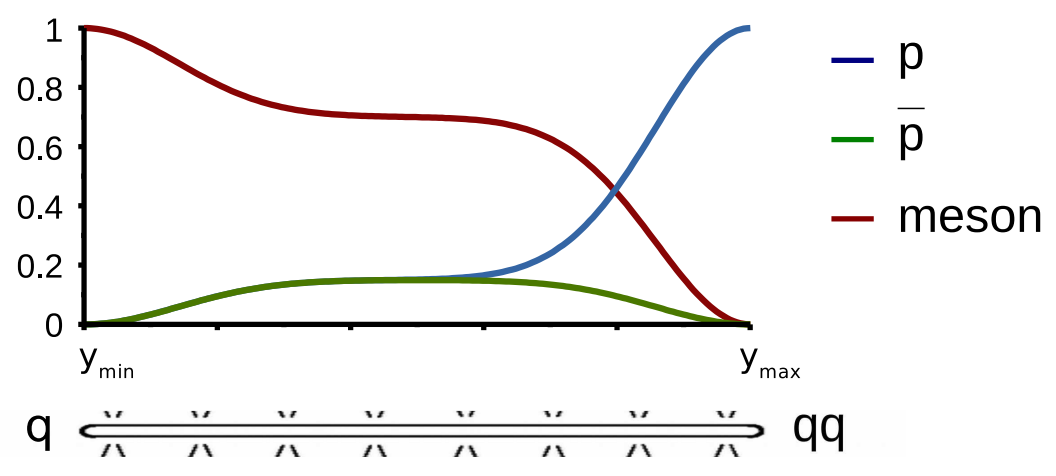

Figure 1: A sketch of a particle composition, coming from one string: fractions of protons, antiprotons and mesons as a function of rapidity.

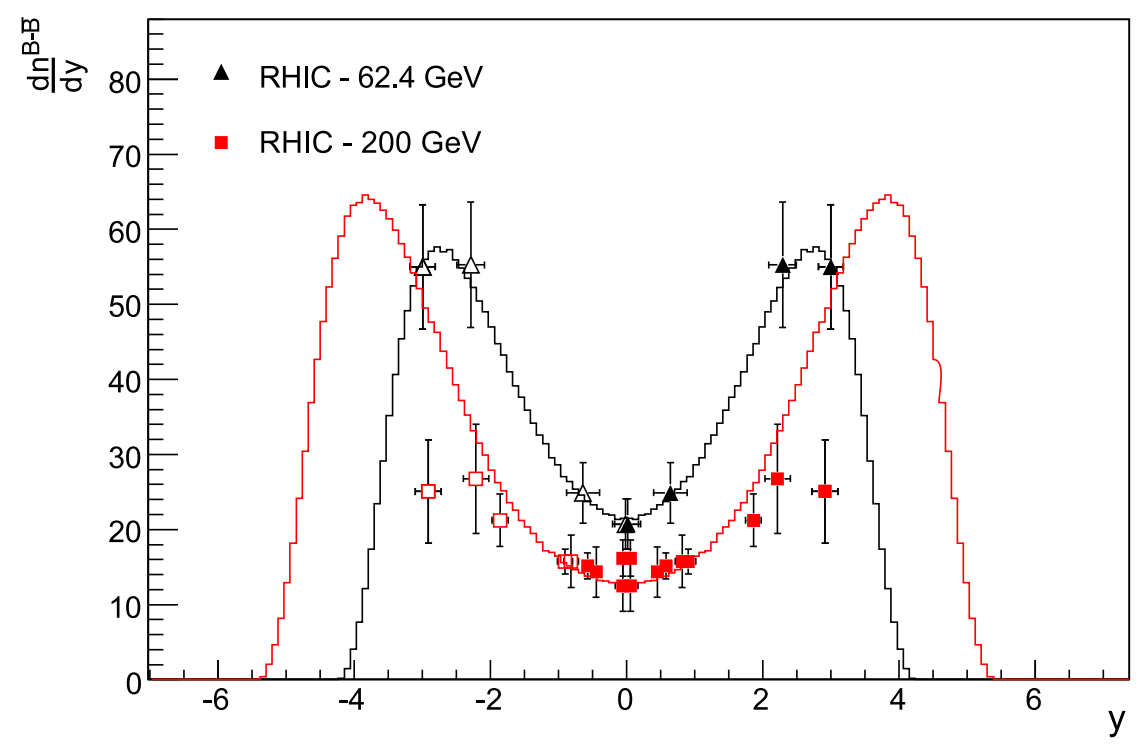

Figure 2: Net baryon rapidity distribution in $\mathrm{Au}+\mathrm{Au}$ collisions. Points - experimental data at RHIC, lines results of calculations in a valence string model [37]. 


\section{Long-range correlations}

The long-range correlations between observables in two separated rapidity windows (backward $B$ and forward $F$ ) are numerically characterized by correlation functions and correlation coefficients. The correlation function is defined as an average of the observable on backward window at fixed value of the observable in the forward window:

$$
f(F)=\langle B\rangle_{F} .
$$

Both $B$ and $F$ could be either $N_{\mathrm{ch}}$ - the number of charged particles in the rapidity window or $p_{t}$ - mean in the event transverse momentum of charged particles in the given window:

$$
p_{t}=\frac{1}{N_{\mathrm{ch}}} \sum_{i=1}^{N_{\mathrm{ch}}} p_{t i}
$$

Correspondingly, tree types of correlation functions are defined [35, 36]: $n-n\left(f_{n-n}\right)$, pt-n $\left(f_{\mathrm{p}_{\mathrm{t}}}-\mathrm{n}\right)$ and pt-pt $\left(f_{\mathrm{p}_{\mathrm{t}}}-\mathrm{p}_{\mathrm{t}}\right)$.

$$
f_{\mathrm{n}-\mathrm{n}}\left(n_{F}\right)=\left\langle n_{B}\right\rangle_{n_{F}}, \quad f_{\mathrm{p}_{\mathrm{t}}-\mathrm{n}}\left(n_{F}\right)=\left\langle p_{t B}\right\rangle_{n_{F}}, \quad f_{\mathrm{p}_{\mathrm{t}}-\mathrm{p}_{\mathrm{t}}}\left(n_{F}\right)=\left\langle p_{t B}\right\rangle_{p_{t F}} .
$$

The correlation coefficient represents the slope of the correlation function:

$$
b_{B-F}=\left.\frac{d f(F)}{d F}\right|_{F=<F>} .
$$

In order to reduce experimental and theoretical uncertainties, it is often useful to switch to normalized variables: $B \rightarrow B /\langle B\rangle, F \rightarrow F /\langle F\rangle$. In this case, the $p_{t}-n$ correlation coefficient becomes dimensionless, and also both $n-n$ and $p_{t}-p_{t}$ correlation coefficients do not change in case of symmetrical rapidity windows. In normalized variables:

$$
\begin{aligned}
b_{n n}= & \left.\frac{<n_{F}>}{<n_{B}>} \cdot \frac{d<n_{B}>}{d n_{F}}\right|_{n_{F}=<n_{F}>}, \\
b_{p_{t}-n}= & \left.\frac{<n_{F}>}{<p_{t_{B}}>} \cdot \frac{d<p_{t_{B}}>}{d n_{F}}\right|_{n_{F}=<n_{F}>}, \\
b_{p_{t}-p_{t}}= & \left.\frac{<p_{t F}>}{<p_{t_{B}}>} \cdot \frac{d<p_{t_{B}}>}{d p_{t_{F}}}\right|_{p_{t F}=<p_{t F}>} .
\end{aligned}
$$

There is an alternative definition on long-range correlation coefficient [38]: $b_{\text {corr }}=\frac{\left\langle n_{B} n_{F}\right\rangle-\left\langle n_{B}\right\rangle\left\langle n_{F}\right\rangle}{\left\langle n_{F}^{2}\right\rangle-\left\langle n_{F}\right\rangle^{2}}$. The definitions are equivalent in case of linear correlation functions, and both of them are used in theoretical and experimental studies [39, 40, 41, 42].

In our calculations we took $\mathrm{p}+\mathrm{p},{ }^{7} \mathrm{Be}+{ }^{9} \mathrm{Be}, \mathrm{p}+\mathrm{Pb}, \mathrm{Ar}+\mathrm{Ca}, \mathrm{Au}+\mathrm{Au}$ and $\mathrm{Pb}+\mathrm{Pb}$ collisions at the colliding energies $\sqrt{s}$ from $5 \mathrm{GeV}$ to $62.4 \mathrm{GeV}$. The set of the colliding systems and energy range have been selected in accordance with the experimental program of NA61 Collaboration, BESII program at RHIC and future detectors at FAIR and NICA facilities.

For $\mathrm{p}+\mathrm{p}, \mathrm{Be}+\mathrm{Be}, \mathrm{p}+\mathrm{Pb}$ collisions minimum bias events have been taken; for heavier species $(\mathrm{Ar}+\mathrm{Ca}, \mathrm{Au}+\mathrm{Au}, \mathrm{Pb}+\mathrm{Pb})$ two centrality classes have been considered: the central events, which correspond to the number of participant nucleons $N_{\text {part }}>(A+B) / 2$, and the peripheral ones, with $N_{\text {part }} \leq(A+B) / 2$. Here, $A$ and $B$ are mass numbers of the colliding nuclei. 
We selected three rapidity windows configurations:

$$
\begin{aligned}
& (-1 ; 0)-(0 ; 1) ; \\
& (0 ; 1)-(1 ; 2) ; \\
& (0 ; 1)-(2 ; 3) .
\end{aligned}
$$

These windows correspond to mid-rapidity configuration (first two) and middle-forward case (third one). The forward window in the third configuration probes the rapidity region, where significant net baryon density is expected (see Fig. 2).

\section{Results}

\subsection{Correlation functions}

Fig. 3 shows all three types of correlation functions. The n-n correlation function is obtained to be close to linear at low values of $n_{F}$, which correspond to peripheral collisions. The slope of the correlation function decreases with $n_{F}$ and tends to saturate at high values of $n_{F}$. The results on pt-n correlations demonstrate small positive pt-n slope of pt-n correlation function. The ptpt correlation function is found strongly non-linear in minimum bias collisions. The predictions are similar to the ones, obtained for $\mathrm{Pb}+\mathrm{Pb}$ collisions at the SPS energy in string fusion approach $[35,36]$ and experimental data of the NA49 experiment [43].
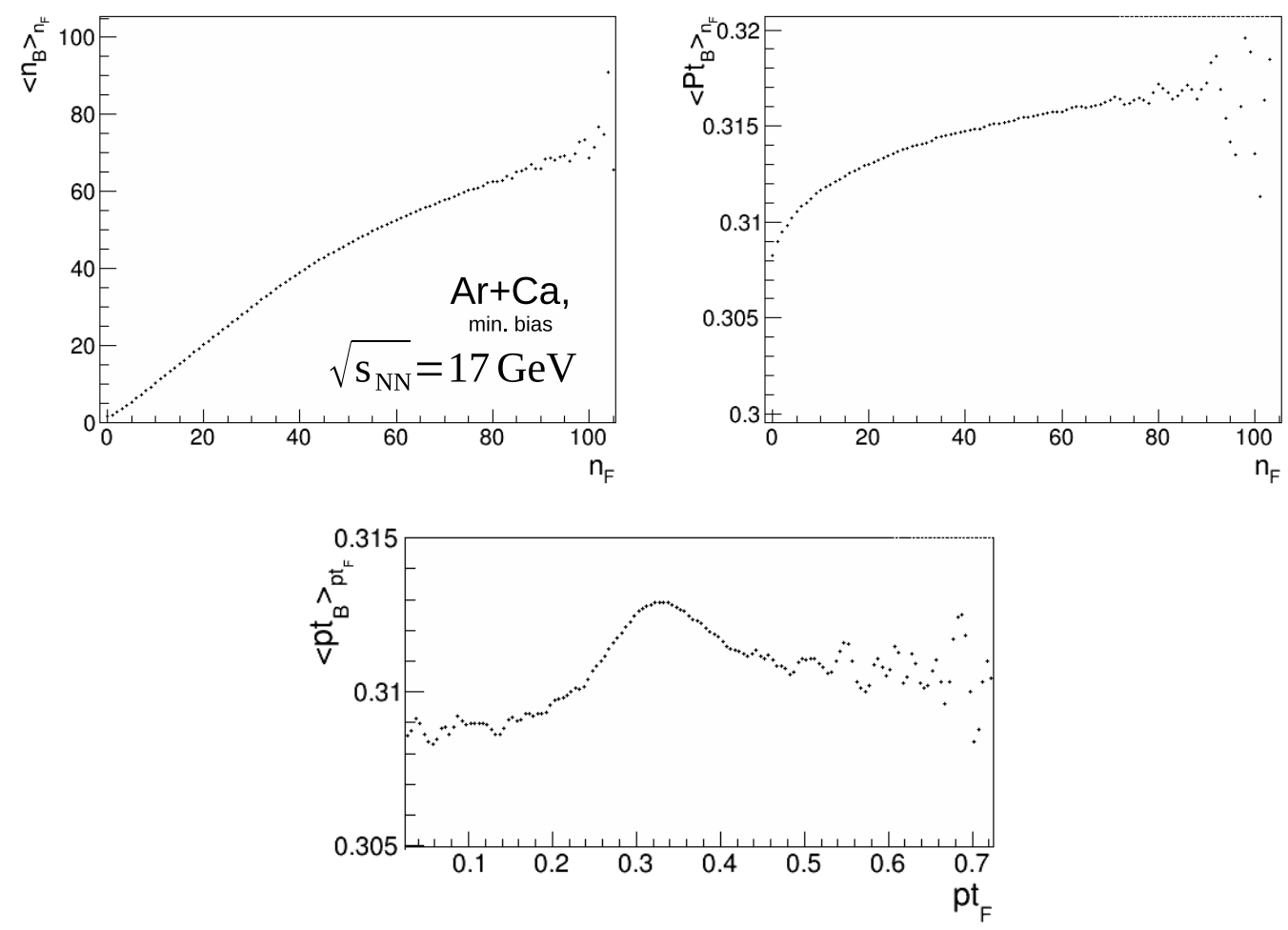

Figure 3: Correlation functions in $\mathrm{Ar}+\mathrm{CA}$ collisions at $\sqrt{s_{N N}}=17 \mathrm{GeV}$ : $\mathrm{n}-\mathrm{n}$ correlation (left), pt-n correlation (right) and pt-pt correlation (bottom). Rapidity windows configuration is $(-1 ; 0),(0 ; 1)$. 


\subsection{Correlation coefficients}

In the Fig. 4 the multiplicity-multiplicity correlation coefficient in proton-proton collisions is plotted as a function of the center-of-mass collision energy per nucleon $\sqrt{s_{N N}}$. The results demonstrate monotonic increase of the $b_{\mathrm{n}-\mathrm{n}}$ with $\sqrt{s}$.

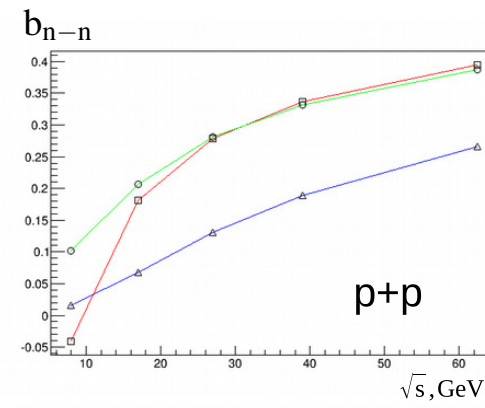

$$
\begin{aligned}
& -(-1 ; 0),(0 ; 1) \\
& -(0 ; 1),(1 ; 2) \\
& \leftarrow(0 ; 1),(2 ; 3)
\end{aligned}
$$
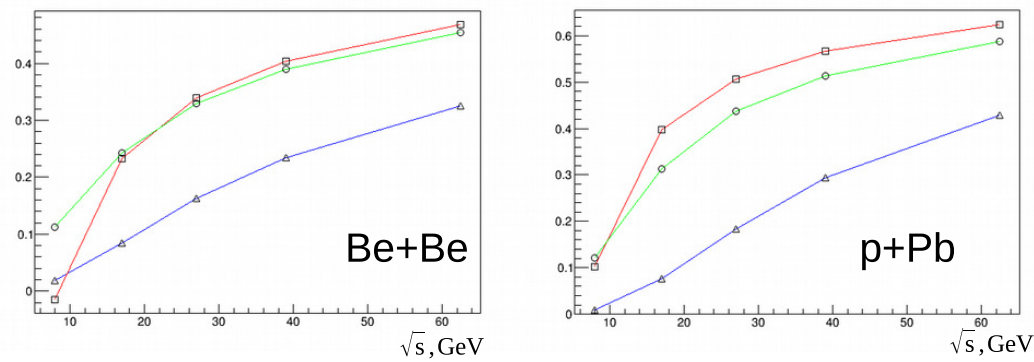

Figure 4: Dependence of $n-n$ correlation coefficient on the collision energy in proton-proton, $\mathrm{Be}+\mathrm{Be}$ and $\mathrm{p}+\mathrm{Pb}$ collisions. Three rapidity windows configurations are represented by different colors.
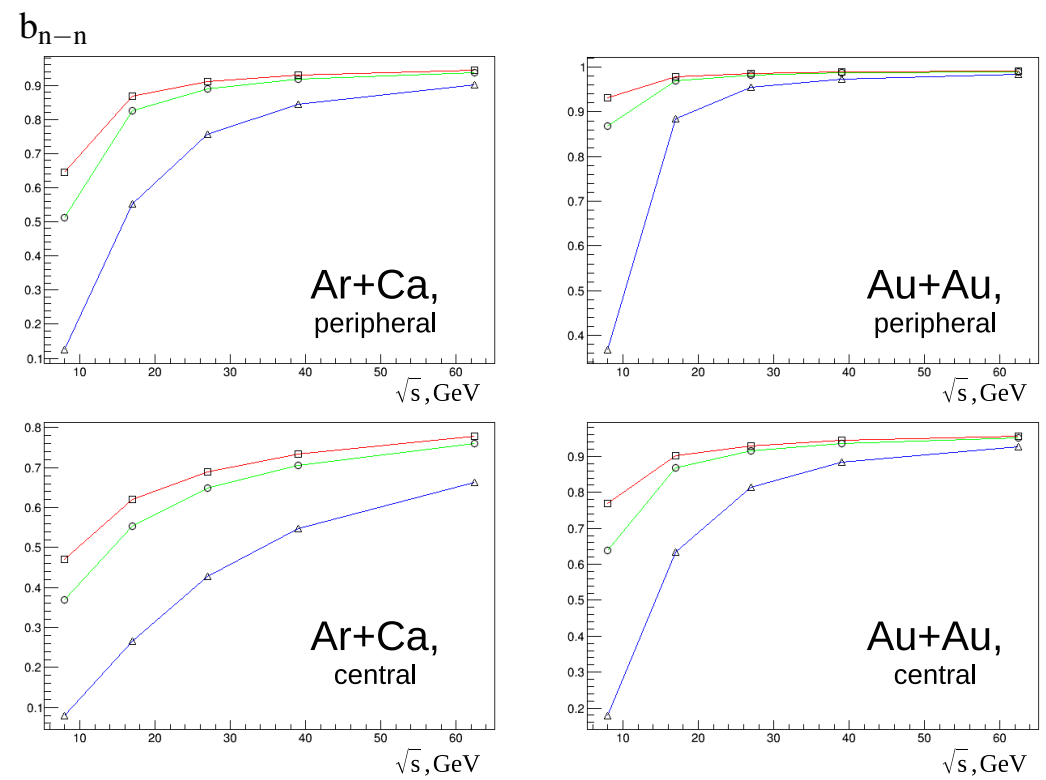

Figure 5: Dependence of $n-n$ correlation coefficient in three rapidity windows configurations on the collision energy in $\mathrm{Ar}+\mathrm{Ca}$ and $\mathrm{Au}+\mathrm{Au}$ collisions. The color notation is the same as in Fig. 4. 
In general, the n-n correlation coefficient is higher in mid-rapidity and decrease going from mid-rapidity to middle-forward configuration. Such behaviour is in agreement with the previous studies [30, 44, 45], where it was shown that $b_{\mathrm{n}-\mathrm{n}}$ decreases with an increase of the rapidity gap and going to the forward rapidity region.

The results on n-n correlations in proton-ion and light ion collisions are also shown in Fig. 4. The correlation coefficient is significantly higher than the one in pp collisions, but no saturation with the energy is achieved.

Multiplicity-multiplicity correlation coefficients for heavier ion species are plotted in Fig. 5. The results are split into two different centrality classes, as described above. The $\mathrm{Pb}+\mathrm{Pb}$ collisions, which have a similar pattern as $\mathrm{Au}+\mathrm{Au}$, are not shown. It is found, that the $b_{\mathrm{n}-\mathrm{n}}$ is less in the central class than in the peripheral one. Such behaviour is in a correspondence with the string fusion predictions [44]. This fact is also in agreement with a saturation trend of the correlation function (Fig. 3). However, one should keep in mind that this observable is subject to the centrality class width dependence [44], and the central class in our calculations seems to be narrower. So, in order to provide the more convincing predictions, the calculations in narrow centrality classes are required.
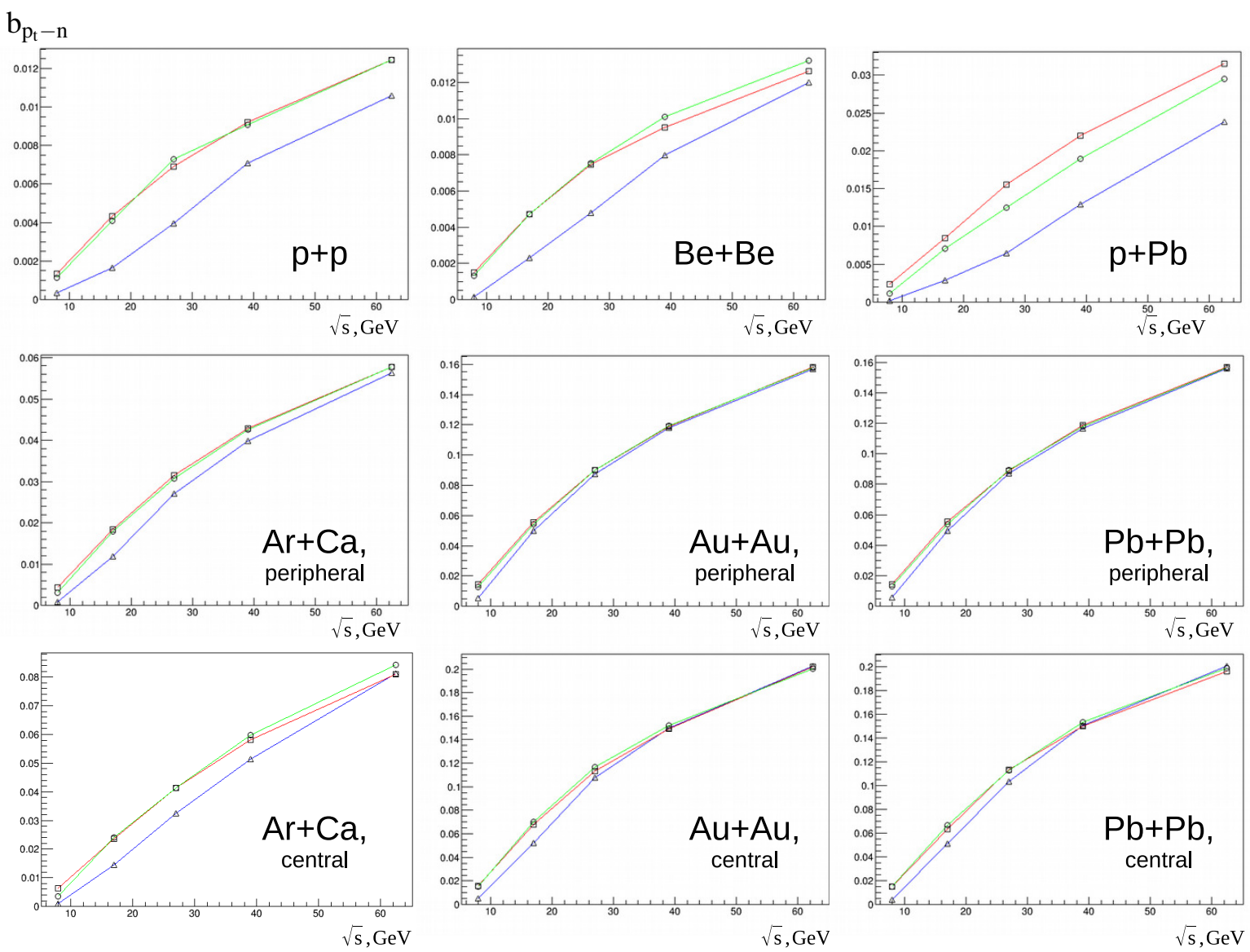

Figure 6: Dependence of pt-n correlation coefficient in three rapidity windows configurations on the collision energy in various colliding systems. The color notation is the same as in Fig. 4. 
The results on pt-n correlation coefficient are shown in Fig. 6. We find the smooth monotonic behaviour of $b_{\mathrm{pt}-\mathrm{n}}$ with the collision energy, similar to the one, observed for multiplicity correlations. We should note, that at minimal energy, the pt-n correlation is practically zero, which is not true for $n-n$ correlations. It may be related to the fact that $n-n$ correlations are present also in the absence of any collectivity, due to the fluctuations in the number of the emitting sources [46]. In contrary, the pt-n correlations appear only in the presence of the string fusion, and their sign depends on the variance of the initial sources [47]. We should note that the magnitude of the pt-n correlations increases significantly from light to heavier nuclei. However, this observable also suffering from the dependency on the centrality selection [44], so a more deep centrality investigation would improve the calculations.
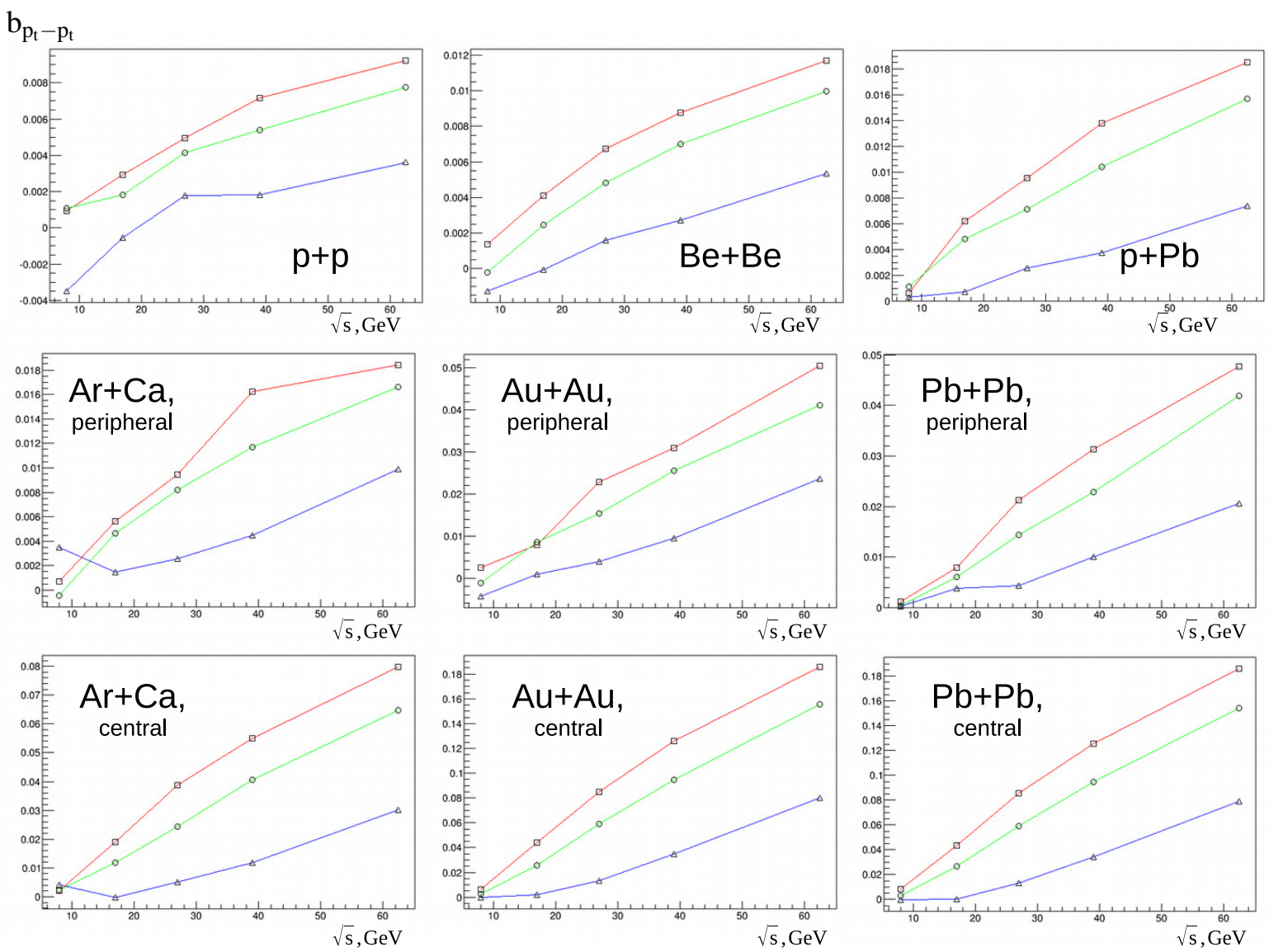

Figure 7: Dependence of pt-pt correlation coefficient in three rapidity windows configurations on the collision energy in various colliding systems. The color notation is the same as in Fig. 4.

Fig. 7 shows the energy dependence of the transverse momentum correlation coefficient. Contrary to the previously discussed observables, $b_{\mathrm{pt}-\mathrm{pt}}$ is a correlation between two intensive variables [36], and it is more robust towards the centrality selection, because it is less influenced by the volume fluctuations due to variation in participant nucleons number and impact parameter. 
In the results of the calculation of $b_{\mathrm{pt}-\mathrm{pt}}$ one observes a monotonic increase with the collision energy mostly. However, in $\mathrm{Ar}+\mathrm{Ca}$ there is a non-monotonic dependence with a minimum around $\sqrt{s}=17 \mathrm{GeV}$. One should stress that the pt-pt correlation coefficient behaves non-monotonically only in case of middle-forward rapidity configurations, while in the other two cases (midrapidity ones) the dependence is smooth. This picture suggests the existence of a phase transition in the area of forward rapidity (note that the larger rapidity corresponds to higher baryon chemical potential) with smooth crossover in midrapidity (where the baryon density is minimal). Note that similar nonmonotonic behavior has been obtained experimentally in the energy range $10-20 \mathrm{GeV}$ for different fluctuational observables (for example, the deviations from Poisson of net-proton fluctuations at RHIC [48]), and it was attributed to the existence of the phase transition and the critical point on the QCD phase diagram. In the Fig. 8 we show a more detailed study of pt-pt correlations in $\mathrm{Ar}+\mathrm{Ca}$ collisions in the energy range between 5 and $20 \mathrm{GeV}$.
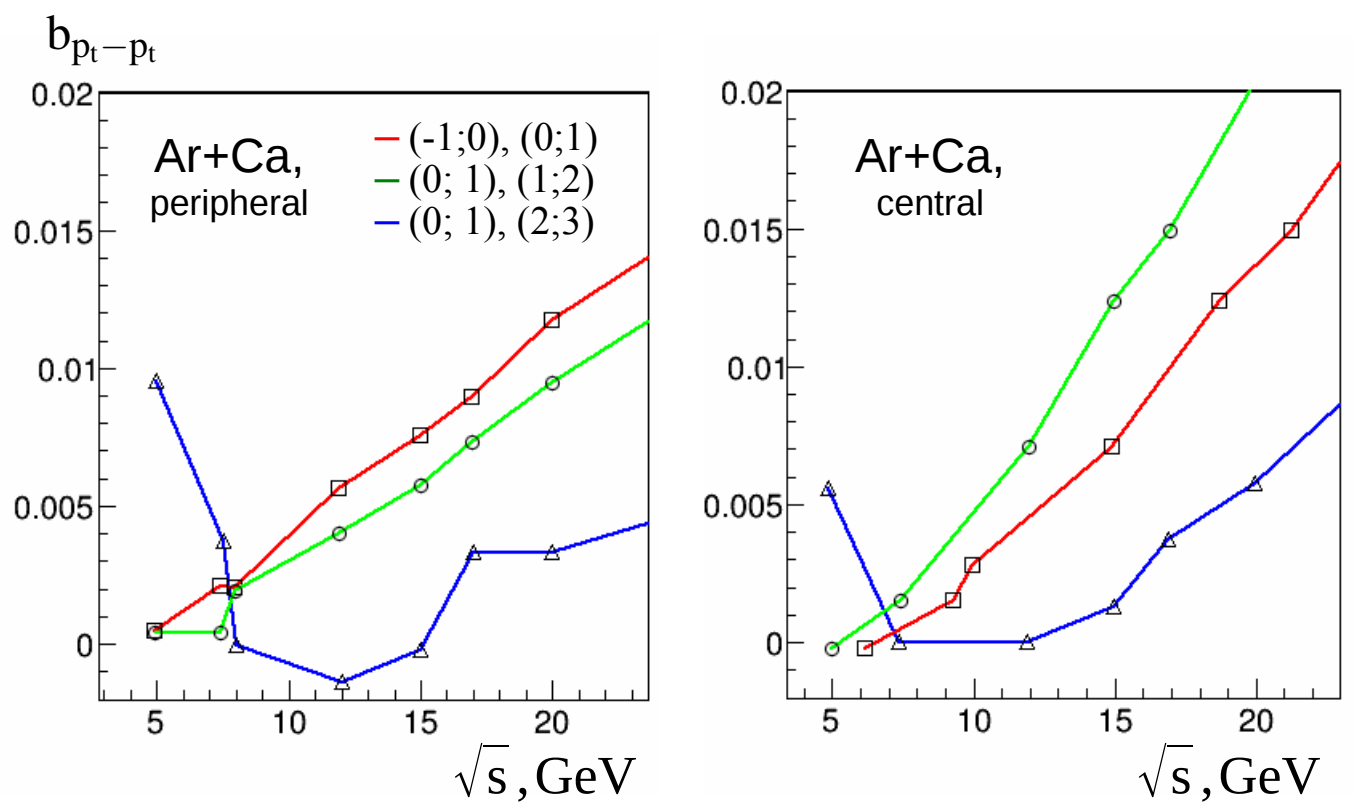

Figure 8: Dependence of pt-pt correlation coefficient in three rapidity windows configurations on the collision energy in $\mathrm{Ar}+\mathrm{Ca}$ collisions.

We performed these addition calculations in order to study the shape of the observed dip in ptpt correlation energy dependence. The results confirmed previous calculation. The central position of the dip in peripheral collisions is found at higher energy than in central, which suggests that the phase transition happens earlier in more central collisions. Overall, the obtained picture advises, that being applied to the range of non-zero baryon chemical potential, the present model, based on the string fusion approach, reproduces the phase transition one expects in the QCD equation of state.

The calculations highlight the importance of Ar+Ca run in the beam energy and system size scan by the NA61 Collaboration at the SPS scheduled on 2015 year [49]. The experimental study of the long-range pt-pt correlations, as well as different strongly intensive observables [50, 51], would be crucial for the improvement of our understanding of the phase diagram of the strongly interacting matter. 


\section{Conclusions}

String fusion approach to the quark-gluon plasma formation at non-zero baryon chemical potential has been proposed. A model for the string fusion, accounting finite rapidity width of strings, for pp, pA and AA collisions is developed and applied at the SPS energies. Long-range correlation functions and coefficients are studied. Smooth monotonic behavior of $n-n$ and pt-n correlation with energy with non-monotonic pt-pt correlation dependence in $\mathrm{Ar}+\mathrm{Ca}$ collisions have been obtained. A more detailed scan, including calculation of correlations in narrow centrality classes, is required, as well as an extension of the model for net-charge and net-proton fluctuation and correlation studies, exploring the strongly intensive variables.

\section{Acknowledgements}

V. Kovalenko acknowledges Saint-Petersburg State University for the research grant 11.38.193.2014 and Special Rector's Scholarship. He is also grateful to the Dynasty Foundation.

\section{References}

[1] Y. Aoki, G. Endrodi, Z. Fodor, S. D. Katz, and K. K. Szabo, The order of the quantum chromodynamics transition predicted by the standard model of particle physics, Nature 443, 675 (2006) [hep-lat/0611014].

[2] C. Blume, Studies on the QCD Phase Diagram at SPS and FAIR, J. Phys. Conf. Ser. 422, 012022 (2013).

[3] C. Schmidt, Lattice QCD at finite density, POS (LAT2006) 021 (2006) [hep-lat/0610116].

[4] O. Philipsen, Lattice calculations at non zero chemical potential, PoS (ConfinementVI I ) 0111 (2009).

[5] F. R. Brown, et al., On the existence of a phase transition for QCD with three light quarks, Phys. Rev. Lett. 65, 2491 (1990).

[6] A. A. Khan, et al., Phase structure and critical temperature of two-flavor QCD with a renormalization group improved gauge action and clover improved Wilson quark action, Phys. Rev. D 63, 034502 (2001) [hep-lat/0008011].

[7] Z. Fodor, S. D. Katz, Critical point of QCD at finite T and $\mu$, lattice results for physical quark masses, JHEP 0404, 050 (2004) [hep-lat/ 0402006 ] .

[8] P. de Forcrand and O. Philipsen, The chiral critical line of $N_{f}=2+1$ QCD at zero and non-zero baryon density, JHEP 01, 077 (2007) [hep-lat/ 0607017 ]

[9] O. Philipsen, Status of the QCD Phase Diagram from Lattice Calculations, Acta Phys. Polon. Supp. 5, 825-835 (2012) [arXiv:1111.5370 [hep-ph] ].

[10] M. Gazdzicki, Onset of Deconfinement and Critical Point: NA49 and NA61/SHINE at the CERN SPS, Eur. Phys. J. ST 155, 37 (2008) [arXiv:0801.4919 [nucl-ex] ] .

[11] C. Hohne, Physics of compressed baryonic matter, J. Phys. Conf. Ser. 420, 012016 (2013). 
[12] M. Gazdzicki (NA49 and NA61/SHINE Collaborations), NA49/NA61: results and plans on beam energy and system size scan at the CERN SPS, J. Phys. G 38, 124024 (2011) [arXiv: 1107.2345 [nucl-ex] ].

[13] G. Odyniec, The RHIC Beam Energy Scan program in STAR and what's next ..., J. Phys. Conf. Ser. 455, 012037 (2013).

[14] P. Staszel, CBM experiment at FAIR, Acta Phys. Pol. B 41, 341 (2010).

[15] V. Toneev, The NICA/MPD project at JINR (Dubna), POS (CPOD 07 ) 057 (2007) [arXiv:0709.1459 [nucl-ex] ].

[16] M. S. Borysova, Quark-gluon plasma signals in CBM experiment, J. Phys. Studies 14, 3203 (2010).

[17] N. Antoniou, et al. (NA61 Collaboration). Study of hadron production in hadron-nucleus and nucleus-nucleus collisions at the CERN SPS. SPSC-P-330, CERN-SPSC-2006-034 (2006).

[18] M. A. Braun, C. Pajares, J. Ranft, Fusion of strings vs. percolation and the transition to the quark-gluon plasma, Int. J. Mod. Phys. A 142689 (1999).

[19] N. S. Amelin, M. A. Braun, C. Pajares, Multiple production in the Monte Carlo string fusion model, Phys. Lett. B 306, 312 (1993).

[20] M. A. Braun and C. Pajares, Particle production in nuclear collisions and string interactions, Phys. Lett. B 287, 154 (1992).

[21] M. A. Braun and C. Pajares, A Probabilistic model of interacting strings, Nucl. Phys. B 390, 542 (1993).

[22] N. S. Amelin, N. Armesto, M.A. Braun, E. G. Ferreiro, and C. Pajares, Long and short range correlations and the search of the quark gluon plasma, Phys. Rev. Lett. 73, 2813 (1994).

[23] O. Kochebina, G. Feofilov, Onset of "ridge phenomenon" in AA and pp collisions and percolation string model, in Proc. XX Baldin ISHEPP (2010) [arXiv:1012.0173 [hep-ph] ] .

[24] M. A. Braun, C. Pajares, V. V. Vechernin, Anisotropic flows from colour strings: Monte Carlo simulations, Nucl. Phys. A 906, 14 (2013) [arXiv:1204.5829 [hep-ph] ].

[25] R. P. Scharenberg, B. K. Srivastava, A. S. Hirsch, Percolation of color sources and the equation of state of QGP in central Au-Au collisions at $\sqrt{s_{N N}}=200 \mathrm{GeV}$, Eur. Phys. J. C 71, 1510 (2011) [arXiv:1006.3260 [nucl-ex]].

[26] J. Dias de Deus, A. S. Hirsch, C. Pajares, R. P. Scharenberg, B. K. Srivastava, Percolation of Color Sources and the Shear Viscosity of the QGP in Central A-A Collisions at RHIC and LHC Energies, arXiv:1106.4271 [nucl-ex] (2011).

[27] B. K. Srivastava, Percolation Approach to Initial Stage Effects in High Energy Collisions, Nucl. Phys. A 926, 142 (2014) [arXiv:1402.2306 [nucl-ex] ] .

[28] J. Dias de Deus, A. S. Hirsch, C. Pajares, R. P. Scharenberg, and B. K. Srivastava, Clustering of color sources and the shear viscosity of the QGP in heavy ion collisions at RHIC and LHC energies, Eur. Phys. J. C 72, 2123 (2012).

[29] V. N. Kovalenko, Modeling of exclusive parton distributions and long-range rapidity correlations in proton-proton collisions at the LHC energies, Phys. Atom. Nucl. 76, 1189 (2013) [arXiv:1211.6209 [hep-ph]].

[30] V. Kovalenko, V. Vechernin, Model of pp and AA collisions for the description of long-range correlations, PoS (Baldin ISHEPP XXI) 077 (2012) [arXiv:1212.2590 [nucl-th]]. 
[31] H. De Vries, C. W. De Jager, and C. De Vries, Atom. Data Nucl. Data Tabl. 36, 495 (1987).

[32] C. Flensburg, G. Gustafson, and L. Lonnblad, Eur. Phys. J. C 60, 233 (2009).

[33] G. Gustafson, Acta Phys. Polon. B 40, 1981 (2009).

[34] V. Kovalenko, Monte Carlo model for pp, pA and AA collisions at high energy: parameters tuning and results, POS (QFTHEP 2013) 052 (2013).

[35] V. V. Vechernin and R. S. Kolevatov, Long-range correlations between transverse momenta of charged particles produced in relativistic nucleus-nucleus collisions, Phys. Atom. Nucl. 70, 1809 (2007) [Yad. Fiz. 70, 1858 (2007)].

[36] V. V. Vechernin and R. S. Kolevatov, On multiplicity and transverse-momentum correlations in collisions of ultrarelativistic ions, Phys. Atom. Nucl. 70, 1797 (2007) [Yad. Fiz. 70, 1846 (2007)].

[37] J. Alvarez-Muniz, R. Conceicao, J. Dias de Deus, M. C. Espirito Santo, J. G. Milhano and M. Pimenta, A Model for net-baryon rapidity distribution, Eur. Phys. J. C 61391 (2009) [arXiv:0903.0957 [hep-ph] ].

[38] J. Adam et al. (ALICE Collaboration), Forward-backward multiplicity correlations in pp collisions at $\sqrt{s}=0.9,2.76$ and $7 \mathrm{TeV}$, arXiv:1502.00230 [nucl-ex] (2015).

[39] A. Capella and J. Tran Thanh Van, "Long Range Rapidity Correlations in Hadron - Nucleus Interactions,” Phys. Rev. D 29, 2512 (1984).

[40] T. Alexopoulos et al. (E735 Collaboration), Charged particle multiplicity correlations in $\overline{\mathrm{p}}$ collisions at $\sqrt{s}=0.3$ TeV to 1.8 TeV, Phys. Lett. B 353, 155-160 (1995).

[41] K. Alpgard et al. (UA5 Collaboration), Forward-Backward Multiplicity Correlations in p anti-p Collisions at 540-GeV, Phys. Lett. B 123, 361 (1983).

[42] G. Aad et al. (ATLAS Collaboration), Forward-backward correlations and charged-particle azimuthal distributions in pp interactions using the ATLAS detector, JHEP 1207, 019 (2012) [arXiv:1203.3100 [hep-ex]].

[43] C. Alt et al. (NA49 Collaboration) and G. A. Feofilov et al. (SPbSU group), Long-range correlations in $\mathrm{PbPb}$ collisions at $158 \mathrm{~A} * \mathrm{GeV}$, in Proc. Relativistic Nuclear Physics and Quantum Chromodynamics, Ed. by A. N. Sissakian, V. V. Burov, and A. I. Malakhov (JINR, Dubna, 2005), Vol. 1, p. 222.

[44] V. Kovalenko and V. Vechernin, Long-range rapidity correlations in high energy AA collisions in Monte Carlo model with string fusion, Eur. Phys. J. Web of Conf. 66, 04015 (2014) [arXiv:1308.6618 [nucl-th]].

[45] V. Kovalenko and V. Vechernin, Forward-backward multiplicity correlations in pp collisions at high energy in Monte Carlo model with string fusion, arXiv:1410.3884 [hep-ph](2014).

[46] V. V. Vechernin, Correlations between multiplicities in rapidity and azimuthally separated windows, arXiv:1210.7588 [hep-ph] (2012).

[47] E. Andronov, V. Vechernin, The correlation between transverse momentum and multiplicity of charged particles in a two-component model, POS (QFTHEP 2013 ) 054 (2014).

[48] L. Adamczyk, et al. (STAR Collaboration), Beam energy dependence of moments of the net-charge multiplicity distributions in Au+Au collisions at RHIC, Phys. Rev. Lett. 113, 092301 (2014) [arXiv:1402.1558 [nucl-ex]]. 
[49] D. Kuchler, M. O`Neil, R. Scrivens and R. Thomae, Preparation of a primary argon beam for the CERN fixed target physics, Rev. Sci. Instrum. 85, 02A954 (2014).

[50] M. Gazdzicki, M. I. Gorenstein and M. Mackowiak-Pawlowska, Normalization of strongly intensive quantities, Phys. Rev. C 88, 024907 (2013) [arXiv:1303.0871 [nucl-th] ].

[51] M. I. Gorenstein and K. Grebieszkow, Strongly Intensive Measures for Transverse Momentum and Particle Number Fluctuations, Phys. Rev. C 89, 034903 (2014) [arXiv: 1309.7878 [nucl-th] ]. 